\title{
Autoconcepto de Género y Sistema de Valores
}

\author{
ESTER BARBERÁ \\ LUIS MAYOR \\ Universidad de Valencia

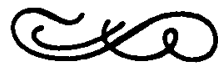

\begin{abstract}
Resumen
El presente trabajo tiene por objeto estudiar los correlatos de valor asociados a los constructos psicológicos de masculinidad y feminidad. Para ello, se han relacionado las puntuaciones obtenidas por una muestra de ambos sexos en las escalas de masculinidad (M), feminidad $(F)$, y masculinidad-femenidad (M-F), del Earlier Personal Attributes Questionnaire, de Spence y Helmreich (1978), con las prioridades establecidas por los mismos sujetos en los valores terminales e instrumentales del Rockeab Value Survey (Rokeach, 1973). La asociación que muestran los resultados entre el autoconcepto de género y los sistemas de valores reafirma la conocida interpretación, según la cual los valores de carácter agente/instrumental se hallan vinculados a la masculinidad, y los comunallexpresivos a la feminidad. Se añaden otras consideraciones acerca del género y los valores relativas a la edad. Estos resultados se discuten, a un nivel más teórico, desde la perspectiva del autoconcepto, corroborando la idea de que las autodescripciones en términos de valores no expresan preferencias objetivas, pretendidamente neutrales, sino que están marcadas por la significación afectiva que tienen para el sujeto.
\end{abstract}

\section{Abstract}

The aim of this article is to study the relation between the structure of values and masculinity and femininity psychological constructs. To this purpose, in a sample using subjects of both sexes, we have related the scores obtained in masculinity $(M)$, feminity $(F)$, and masculinity-feminity $(M-F)$ scales, from the Earlier Personal Attributes Questionnaire (Spence $y$ Helmreich, 1978), with the priorities obtained for the terminal and instrumental value, assessed with the Rockeach Value Survey (Rokeach, 1973). The results of this comparison between the self-concept for gender and the structure of values confirm the general knowledge that the agentic/instrumental values are associated with masculinity, and the communal/expressive ones are associated with femininity. We have also included some considerations about gender and values in relation to age. These results are discussed from the point of view of self-concept. The idea that the evaluative self-descriptions are neither neutral nos objective, but marked by the affective signification they bave for the subject, is reinforced.

Dirección del autor: Facultad de Psicología. Universidad de Valencia. Avda. de Blasco Ibáñez, 46010 Valencia. 


\section{AUTOCONCEPTO DE GENERO Y SISTEMA DE VALORES}

Existen algunos términos, de origen relativamente lejano a la literatura psicológica, cuya reactualización ha ido acompañada de un progresivo enriquecimiento semántico así como de una mayor precisión y rigor conceptual. Entre ellos, sin duda, la noción de «esquema» ocupa un lugar preferencial, habiendo sido calificada de omnipresente para la psicología cognitiva actual (De Vega, 1984). En cuanto entidades conceptuales complejas, "los esquemas» presentan un considerable poder explicativo, de elevada utilidad teórica. En los últimos años, un buen número de autores (Rumelhart y Ortony, 1977; Hamilton, 1981; Higgins, Herman, y Zanna, 1981) se han representado el conocimiento humano a partir de un sistema jerarquizado de esquemas interconectados, coincidiendo en considerar el «autoconcepto" como un tipo básico de esquema social, que en tanto estructura cognitiva específica, parece intervenir en el procesamiento de cualquier modalidad informativa, permitiendo categorizar, explicar y evaluar la experiencia.

Entre los esquemas particulares englobados en el rótulo genérico de «autoconcepto", uno de los que ha merecido un tratamiento más sistemático y exhaustivo, quizás debido a su universalidad y relevancia en la configuración subjetiva, ha sido el «esquema de género". Hasta tal punto la relación del género y el autoconcepto es estrecha, que las ideas y atributos sobre la propia masculinidad o feminidad, así como las conductas específicas en base al sexo, se convierten en aspectos centrales de la identidad personal. S.L.Bem (1981) describe a los sujetos tipificados sexualmente como aquéllos que organizan sus autoconceptos y conductas sobre la idea de género, existiendo evidencia experimental (Markus, Crane, Bernstein y Siladi, 1982; Crane y Markus, 1982) del grado en que los rasgos psicológicos de masculinidad, feminidad, $o$ androginia influyen en la comprensión y adecuación al entorno.

En casi todas las culturas, nacer mujer o nacer varón se convierte, antes que la edad u otra variable relevante, como la estirpe social, en punto de referencia claro de comportamientos, actividades y roles característicos (Barberá, 1983). La socialización al rol del género conlleva el desarrollo de escalas evaluativas distintas que ponen de manifiesto la importancia de aquélla para el desarrollo de una autoimagen ajustada a la normativa social.

Partiendo de trabajos precedentes (Atkinson, 1964; Feather, 1971, 1975), J. Eccles-Parsons ha destacado, desde los presupuestos de valenciaexpectativa, el papel desempeañdo por el sistema axiológico en conductas (de elección) de logro, explicitando el modo en que las necesidades y valores personales vienen determinados por la centralidad que ejercen sobre la autodefinición de un sujeto como varón o mujer. Esto permite reinterpretar, desde una posición menos desfavorecida para las mujeres, la hipótesis del miedo al éxito de Horner (1968) como temor, común a hombres y mujeres, a la desviación de los estándares interiorizados del rol sexual (Anastasi, 1985).

Desde el posicionamiento de Rockeach, el sistema de valores, que se desarrolla muy tempranamente en la vida del sujeto, cumple funciones clave de índole normativa y motivacional al servicio de la autodefensa, la adaptación y la realización personal, que hacen del autoconcepto el epicentro 


\section{3}

de configuración de los demás subsistemas (Barberá y Mayor, 1987). Los esquemas referidos al género conllevarían así dimensiones no sólo descriptivas sino también evaluativas y prescriptivas.

En esta línea, la presente investigación trata de establecer las referencias evaluativas asociadas a la masculinidad y feminidad, poniendo en relación las puntuaciones obtenidas en las escalas $\mathrm{M}$ (masculino), $\mathrm{F}$ (femenino), $\mathrm{y}$ M-F (masculino-femenino), del cuestionario EPAQ (Earlier Personal Attributes Questionnaire) (Spence y Helmreich, 1978; Spence, Helmreich y Holahan, 1979), con las prioridades evaluativas registradas a través del Rokeach Value Survey (RVS) (Rokeach, 1973).

\section{METODO}

\section{Descripción general}

A lo largo del curso $1985-86$ se llevó a cabo la recogida de datos mediante el pase a una muestra compuesta por 174 estudiantes (42 varones y 132 mujeres) con una edad media de veintidós años, de segundo curso de Psicología de la Universidad de Valencia, de las dos pruebas siguientes: el EPAQ de Spence y Helmreich (1978) y el RVS (Rockeach, 1973). La primera, de papel y lápiz, está compuesta por veinticuatro, items representativos de rasgos que describen la propia personalidad, pudiendo optarse en cada uno de ellos entre cinco posibilidades (Nada-A, Algo-B, Normal-C, Bastante-D, Mucho-E) dentro de una escala bipolar. Del total de ítems, ocho integran la escala $\mathrm{M}$ (masculino), ocho la escala $\mathrm{F}$ (femenino), y los ocho restantes la escala M-F (masculino-femenino). Cada sujeto obtiene, pues, tres puntuaciones mediante la suma de la cuantificación asignada a las ocho características de cada escala. Dado que cada uno de los ítems puede llegar a puntuar un máximo de cuatro $(0-1-2-3-4)$ la puntuación más alta posible es de treinta y dos por escala.

El RVS se compone de dos listas con dieciocho opciones cada una de ellas, correspondientes a dos escalas de valores terminales e instrumentales. Los primeros incluyen conceptos que hacen referencia a metas generales o estados finales de existencia, mientras que los instrumentales aluden a formas más concretas de comportamiento. Los sujetos han de ordenar los dieciocho valores integrantes de cada lista, de acuerdo con la importancia que presentan para ellos en cuanto a principios rectores de sus vidas, prioridad que va desde el número uno (el que tiene más importancia) al número dieciocho (el que tiene menos importancia).

Hemos utilizado las traducciones llevadas a cabo por Fernández (1983) para el EPAQ y por Garcés (1985) en el caso del RVS.

\section{Procedimiento y Forma de Análisis}

Las dos escalas se aplicaron de forma colectiva durante la primavera de 1986, en condiciones uniformes para toda la muestra. Antes de empezar cada una de las pruebas, se les leyeron despacio y en voz alta las instrucciones correspondientes, con el fin de asegurar su perfecta comprensión, instándoles a trabajar de forma sincera e independiente, ya que sus respuestas iban a permanecer en el más estricto anonimato. Cada sujeto debía re- 


\section{4}

llenar también unos casilleros en los que se les solicitaba información sobre su edad y sexo.

A los datos obtenidos les hemos aplicado los siguientes análisis: a) Prueba $\mathrm{T}$ de Student, al objeto de establecer diferencias, según el sexo, para las distintas variables. a analizar: masculinidad, feminidad, masculinidad-feminidad, y valores terminales e instrumentales. b) Correlaciones productomomento entre los rangos transformados en puntuaciones $z^{*}$ para cada valor $\mathrm{y}$ las puntuaciones obtenidas en las escalas M, F, y M-F. c) Correlaciones de la edad con todas las variables indicadas (M, F, M-F), valores terminales e instrumentales. d) Análisis Factorial y e) Análisis Discriminantes, para tratar de conocer los valores terminales e instrumentales que más discriminan entre los subgrupos de varones y mujeres, por un lado, y entre los conceptos psicológicos de masculinidad y feminidad, por otro.

\section{RESULTADOS}

\section{Diferencias en género y valores, según los sexos}

En relación al género, sólo aparecen diferencias significativas entre hombres y mujeres en la escala de Masculinidad-Feminidad (M-F), lo que debe entenderse, de acuerdo con la interpretación de Spence y Helmreich (1978), en términos de que mientras la masculinidad (M) y la feminidad (F) se refieren a características estereotipadas para uno o el otro sexo pero en general deseables, la escala de masculinidad-feminidad (M-F) contiene ítems cuya deseabilidad social varía en función del sexo.

En cuanto a los valores terminales, los hombres parecen dar más importancia a la «Igualdad (hermandad, igualdad de oportunidades)", en tanto que las mujeres destacan en «Seguridad Nacional (protección de los ataques)» y "Armonía interna (libre de conflictos internos)». En lo que respecta a valores instrumentales, los varones conceden un peso más fuerte a las variables «Intelectual (inteligente, reflexivo)", «Lógico (consciente, racional)», y «Autocontrolado (discreto, autodisciplinado)». Las mujeres, por su parte, lo hacen con los valores «Educado (correcto, de buenas maneras)», y "Honrado (sincero, verdadero)». (Ver Tabla I).

\section{Autoconcepto de género y sistema de valores. Su relación con la edad}

La Tabla II presenta las correlaciones obtenidas entre las medidas del RVS y las puntuaciones de masculinidad (M), Feminidad (F), y Masculinidad-Feminidad (M-F) en el EPAQ.

Aunque los resultados indican que, en general, los índices de correlación son bajos, cabe hacer las siguientes observaciones: la masculinidad correlaciona positivamente con dos valores terminales, primero con «Vida excitante (una vida activa, estimulante)» y, en segundo lugar, con «Libertad (independencia, libre elección)», y negativamente con "Amistad verdadera (relaciones comunicativas)».

Con los valores instrumentales, la masculinidad presenta correlaciones significativas de signo positivo con las variables «Independiente (confianza

* Siguiendo a Hays (1967); Cohen y Cohen (1975) y Feather (1984) sobre el supuesto de mayor facilidad para discriminar la importancia relativa de los valores en los extremos de la escala. 
TABLA I

Prueba $T$ de Student

\begin{tabular}{|c|c|c|c|c|c|c|c|c|}
\hline & \multirow[b]{3}{*}{$\begin{array}{c}\text { Valor } \\
\text { F }\end{array}$} & \multirow{3}{*}{$\begin{array}{c}\begin{array}{c}\text { Comparación } \\
\text { de varianzas }\end{array} \\
\begin{array}{c}\text { Niv. de } \\
\text { signif. } \\
\text { (Valor } \\
\text { de p) }\end{array}\end{array}$} & \multicolumn{6}{|c|}{ Comparación de medias } \\
\hline & & & & \multicolumn{2}{|c|}{$\begin{array}{l}\text { Varianzas } \\
\text { iguales }\end{array}$} & \multicolumn{3}{|c|}{$\begin{array}{l}\text { Varianzas } \\
\text { distintas }\end{array}$} \\
\hline & & & $\underset{T}{\text { Valor }}$ & $\begin{array}{c}\text { Grados de } \\
\text { libert. }\end{array}$ & $\begin{array}{l}\text { Niv. de } \\
\text { signif. } \\
\text { (Valor } \\
\text { de p) }\end{array}$ & $\underset{T}{\text { Valor }}$ & $\begin{array}{l}\text { Grados de } \\
\text { libert. }\end{array}$ & $\begin{array}{l}\text { Niv. de } \\
\text { signif. } \\
\text { (Valor } \\
\text { de p) }\end{array}$ \\
\hline Masculinidad & 1.24 & 0.426 & -1.48 & 172 & 0.142 & -1.56 & 76.12 & 0.122 \\
\hline Feminidad & 1.21 & 0.492 & 0.99 & 172 & 0.323 & 1.04 & 75.10 & 0.301 \\
\hline Masc.-Fem. & 2.17 & 0.005 & -3.45 & 172 & 0.001 & -4.19 & 102.00 & 0.000 \\
\hline Felicidad & 1.25 & 0.418 & 0.41 & 172 & 0.685 & 0.43 & 76.26 & 0.668 \\
\hline Autorrespeto & 1.14 & 0.640 & 0.16 & 172 & 0.871 & 0.17 & 73.14 & 0.867 \\
\hline Tiempo libre & 1.57 & 0.059 & -1.67 & 172 & 0.096 & -1.49 & 58.54 & 0.142 \\
\hline Salvación & 1.26 & 0.391 & 0.58 & 172 & 0.563 & 0.62 & 76.71 & 0.540 \\
\hline Segur familiar & 1.04 & 0.904 & -0.31 & 172 & 0.755 & -0.32 & 70.30 & 0.753 \\
\hline Satisfac. tarea & 1.20 & 0.433 & -0.30 & 172 & 0.767 & -0.28 & 64.15 & 0.778 \\
\hline Vida confortab. & 1.29 & 0.348 & 0.16 & 172 & 0.872 & 0.17 & 77.51 & 0.864 \\
\hline Amistad verbal & 1.18 & 0.484 & 0.71 & 172 & 0.481 & 0.68 & 64.65 & 0.501 \\
\hline
\end{tabular}




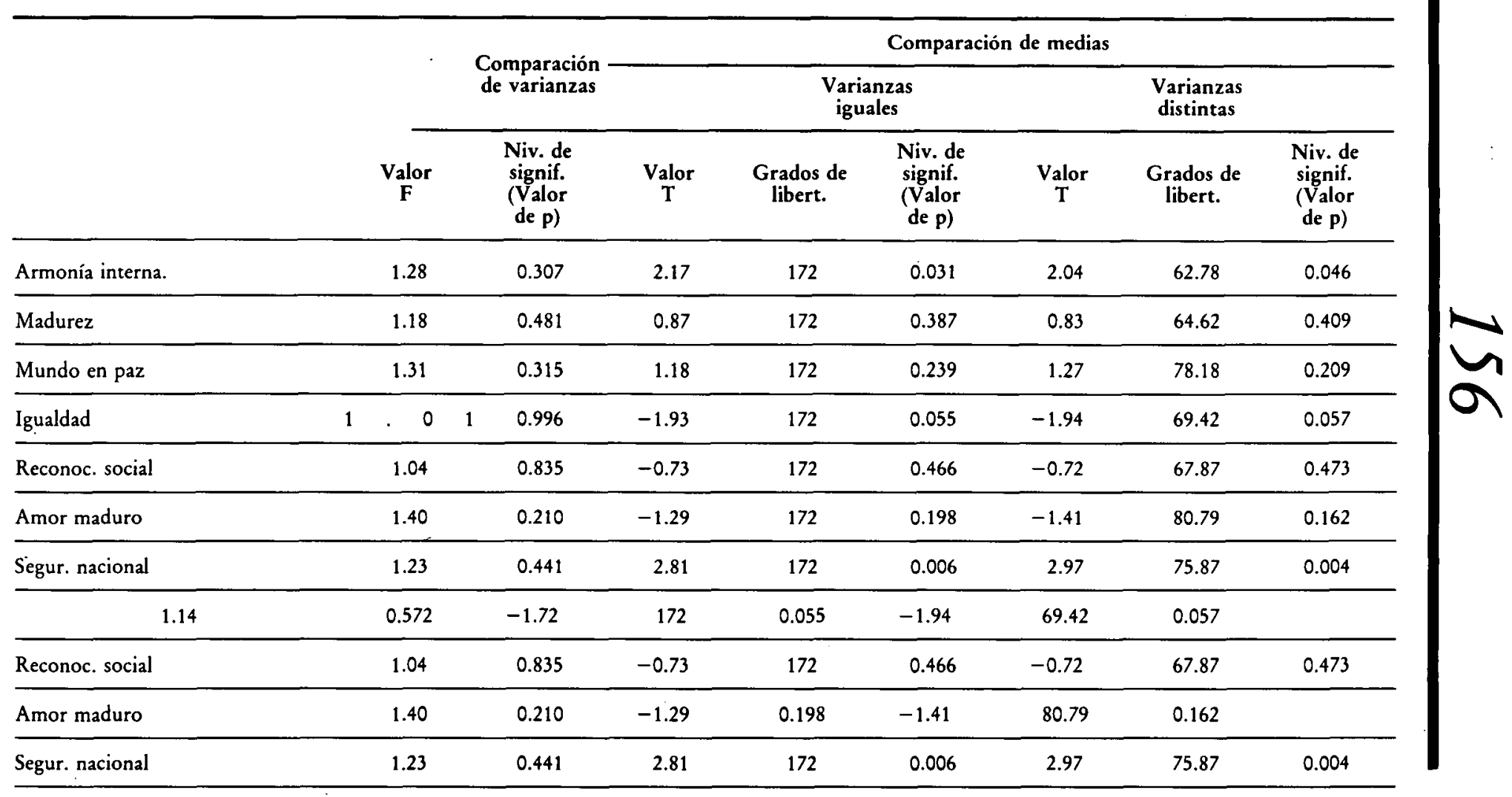


TABla I

(Cont.)

\begin{tabular}{|c|c|c|c|c|c|c|c|c|}
\hline & \multirow[b]{3}{*}{$\begin{array}{l}\text { Valor } \\
\text { F }\end{array}$} & \multirow{3}{*}{$\begin{array}{c}\begin{array}{c}\text { Comparación } \\
\text { de varianzas }\end{array} \\
\begin{array}{c}\text { Niv. de } \\
\text { signif. } \\
\text { (Valor } \\
\text { de p) }\end{array}\end{array}$} & \multicolumn{6}{|c|}{ Comparación de medias } \\
\hline & & & & \multicolumn{2}{|c|}{$\begin{array}{l}\text { Varianzas } \\
\text { iguales }\end{array}$} & \multicolumn{3}{|c|}{$\begin{array}{l}\text { Varianzas } \\
\text { distintas }\end{array}$} \\
\hline & & & $\underset{T}{\text { Valor }}$ & $\begin{array}{l}\text { Grados de } \\
\text { libert. }\end{array}$ & $\begin{array}{l}\text { Niv. de } \\
\text { signif. } \\
\text { (Valor } \\
\text { de p) }\end{array}$ & $\begin{array}{l}\text { Valor } \\
\text { T }\end{array}$ & $\begin{array}{c}\text { Grados de } \\
\text { libert. }\end{array}$ & $\begin{array}{l}\text { Niv. de } \\
\text { signif. } \\
\text { (Valor } \\
\text { de p) }\end{array}$ \\
\hline Mundo de belleza & $\mathrm{i} .14$ & 0.572 & -1.72 & 172 & 0.088 & -1.66 & 65.49 & 0.102 \\
\hline Libertad & 1.16 & 0.602 & -1.50 & 172 & 0.135 & -1.56 & 73.61 & 0.124 \\
\hline Vida excitante & 1.18 & 0.487 & 0.69 & 172 & 0.492 & 0.66 & 64.68 & 0.512 \\
\hline Valiente & 1.03 & 0.886 & -0.46 & 172 & 0.649 & -0.45 & 68.33 & 0.652 \\
\hline Educado & 1.23 & 0.446 & 2.47 & 172 & 0.014 & 2.61 & 75.80 & 0.011 \\
\hline Intelectual & 1.52 & 0.079 & -2.16 & 172 & 0.032 & -1.94 & 59.11 & 0.057 \\
\hline Honrado & 1.17 & 0.562 & 2.78 & 172 & 0.006 & 2.90 & 74.12 & 0.005 \\
\hline Obediente & 1.23 & 0.447 & 0.95 & 172 & 0.346 & 1.00 & 75.78 & 0.321 \\
\hline Lógico & 1.07 & 0.826 & -2.30 & 172 & 0.023 & -2.34 & 71.08 & 0.022 \\
\hline Imaginativo & 1.02 & 0.899 & -1.63 & 172 & 0.104 & -1.62 & 68.45 & 0.109 \\
\hline Capaz. & 1.03 & 0.879 & 0.47 & 172 & 0.637 & 0.47 & 68.27 & 0.640 \\
\hline
\end{tabular}


TABLA I

(Cont.)

\begin{tabular}{|c|c|c|c|c|c|c|c|c|}
\hline & \multirow[b]{3}{*}{$\underset{\text { Valor }}{\mathbf{F}}$} & \multirow{3}{*}{$\begin{array}{c}\begin{array}{c}\text { Comparación } \\
\text { de varianzas }\end{array} \\
\begin{array}{c}\text { Niv. de } \\
\text { signif. } \\
\text { (Valor } \\
\text { de p) }\end{array}\end{array}$} & \multicolumn{6}{|c|}{ Comparación de medias } \\
\hline & & & & \multicolumn{2}{|c|}{$\begin{array}{l}\text { Varianzas } \\
\text { iguales }\end{array}$} & \multicolumn{3}{|c|}{$\begin{array}{l}\text { Varianzas } \\
\text { distintas }\end{array}$} \\
\hline & & & $\underset{T}{\text { Valor }}$ & $\begin{array}{c}\text { Grados de } \\
\text { libert. }\end{array}$ & $\begin{array}{l}\text { Niv. de } \\
\text { signif. } \\
\text { (Valor } \\
\text { de p) }\end{array}$ & $\underset{T}{\text { Valor }}$ & $\begin{array}{c}\text { Grados de } \\
\text { libert. }\end{array}$ & $\begin{array}{l}\text { Niv. de } \\
\text { signif. } \\
\text { (Valor } \\
\text { de p) }\end{array}$ \\
\hline Alegre & 1.01 & 1.000 & 0.67 & $\begin{array}{c}172 \\
172\end{array}$ & 0.502 & 0.67 & 69.30 & 0.503 \\
\hline Autocontrolado & 1.24 & 0.357 & -2.07 & 172 & 0.040 & -1.96 & 63.35 & 0.055 \\
\hline Capaz perdonar & 1.10 & 0.733 & 1.22 & 172 & 0.223 & .1 .25 & 72.08 & 0.214 \\
\hline Capaz de amar & 1.21 & 0.411 & -0.28 & 172 & 0.778 & -0.27 & 63.92 & 0.789 \\
\hline Responsable & 1.33 & 0.295 & 0.90 & 172 & 0.369 & 0.97 & 78.61 & 0.335 \\
\hline Independiente & 1.46 & 0.160 & -1.19 & 172 & 0.235 & -1.31 & 82.49 & 0.193 \\
\hline Limpio & 1.33 & 0.288 & 1.05 & 172 & 0.297 & 1.13 & 78.77 & 0.263 \\
\hline Ambicioso & 1.09 & 0.709 & 1.12 & 172 & 0.263 & 1.10 & 66.74 & 0.276 \\
\hline Liberal & 1.01 & 0.994 & 0.07 & 172 & 0.943 & 0.07 & 69.44 & 0.942 \\
\hline Servicial & 12.30 & 0.341 & 0.00 & 172 & 0.997 & 0.00 & 77.64 & 0.997 \\
\hline
\end{tabular}


TABLA II

Correlaciones producto - momento entre las puntuaciones obtenidas en el EPAQ y el RVS

\begin{tabular}{|c|c|c|c|c|}
\hline $\begin{array}{c}\text { Valores } \\
\text { terminales }\end{array}$ & $\begin{array}{c}\text { Escala } \\
\mathbf{M}\end{array}$ & $\begin{array}{c}\text { Escala } \\
\mathbf{F}\end{array}$ & $\begin{array}{c}\text { Escala } \\
\text { M-F }\end{array}$ & Edad \\
\hline Felicidad & $\begin{array}{l}-.0382 \\
p=.617\end{array}$ & $\begin{array}{r}.0934 \\
p=.220\end{array}$ & $\begin{array}{l}-.0522 \\
p=.494\end{array}$ & $\begin{array}{l}-.1652 \\
\mathrm{p}=.029\end{array}$ \\
\hline Autorrespeto & $\begin{array}{l}-.0121 \\
p=.874\end{array}$ & $\begin{array}{r}.0153 \\
p=.842\end{array}$ & $\begin{array}{r}.0255 \\
\mathrm{p}=.738\end{array}$ & $\begin{array}{c}.0614 \\
p=.421\end{array}$ \\
\hline Tiempo libre & $\begin{array}{l}-.0851 \\
p=.264\end{array}$ & $\begin{array}{l}-.0416 \\
\mathrm{p}=.585\end{array}$ & $\begin{array}{r}.0055 \\
p=.943\end{array}$ & $\begin{array}{r}.1161 \\
p=.127\end{array}$ \\
\hline Salvación & $\begin{array}{l}-.0134 \\
\mathrm{p}=.861\end{array}$ & $\begin{array}{l}-0.518 \\
p=.497\end{array}$ & $\begin{array}{l}-.1400 \\
p=.065\end{array}$ & $\begin{array}{l}-.1447 \\
\mathrm{p}=.052\end{array}$ \\
\hline Seguridad familiar & $\begin{array}{l}-.0369 \\
\mathrm{p}=.629\end{array}$ & $\begin{array}{r}.0848 \\
\mathrm{p}=.266\end{array}$ & $\begin{array}{l}-.1809 \\
p=.017\end{array}$ & $\begin{array}{r}.0492 \\
p=.519\end{array}$ \\
\hline Satisfacción tarea & $\begin{array}{l}-.0859 \\
\mathrm{p}=.259\end{array}$ & $\begin{array}{r}.0368 \\
p=.630\end{array}$ & $\begin{array}{r}.0535 \\
p=.484\end{array}$ & $\begin{array}{l}-.0395 \\
p=.604\end{array}$ \\
\hline Vida confortable & $\begin{array}{l}-.0060 \\
p=.938\end{array}$ & $\begin{array}{r}.0053 \\
\cdot p=.944\end{array}$ & $\begin{array}{r}.0353 \\
\mathrm{p}=.643\end{array}$ & $\begin{array}{l}-.0491 \\
\mathrm{p}=.520\end{array}$ \\
\hline Amistad verdadera & $\begin{array}{l}-.1875 \\
\mathrm{p}=.013\end{array}$ & $\begin{array}{r}.0667 \\
p=.382\end{array}$ & $\begin{array}{l}-.0650 \\
p=.394\end{array}$ & $\begin{array}{l}-.1988 \\
\mathrm{p}=.009\end{array}$ \\
\hline Armonía interna & $\begin{array}{l}-.1016 \\
\mathrm{p}=.182\end{array}$ & $\begin{array}{r}.1659 \\
p=.029\end{array}$ & $\begin{array}{l}-.2224 \\
p=.003\end{array}$ & $\begin{array}{r}.0427 \\
\mathrm{p}=.576\end{array}$ \\
\hline Madurez & $\begin{array}{r}.1005 \\
p=.187\end{array}$ & $\begin{array}{l}-.0505 \\
\mathrm{p}=.508\end{array}$ & $\begin{array}{r}.1404 \\
p=.065\end{array}$ & $\begin{array}{r}.0439 \\
p=.565\end{array}$ \\
\hline Un mundo en paz & $\begin{array}{l}-.0401 \\
\mathrm{p}=.599\end{array}$ & $\begin{array}{r}.0433 \\
\mathrm{p}=.571\end{array}$ & $\begin{array}{l}-.0561 \\
p=.432\end{array}$ & $\begin{array}{r}.0534 \\
p=.484\end{array}$ \\
\hline Igualdad & $\begin{array}{r}.0324 \\
\mathrm{p}=.672\end{array}$ & $\begin{array}{r}.0630 \\
p=.409\end{array}$ & $\begin{array}{r}.0994 \\
\mathrm{p}=.192\end{array}$ & $\begin{array}{r}.1830 \\
\mathrm{p}=.016\end{array}$ \\
\hline Reconocimiento social & $\begin{array}{r}.1252 \\
p=.100\end{array}$ & $\begin{array}{l}-.0568 \\
p=.457\end{array}$ & $\begin{array}{r}.0150 \\
p=.844\end{array}$ & $\begin{array}{l}-.0280 \\
\mathrm{p}=.714\end{array}$ \\
\hline Amor maduro & $\begin{array}{r}.0140 \\
p=.854\end{array}$ & $\begin{array}{l}-.1206 \\
p=.113\end{array}$ & $\begin{array}{r}.0105 \\
p=.891\end{array}$ & $\begin{array}{l}-.0911 \\
\mathrm{p}=.232\end{array}$ \\
\hline Seguridad nacional & $\begin{array}{l}-.0777 \\
p=.308\end{array}$ & $\begin{array}{l}-.0253 \\
p=.741\end{array}$ & $\begin{array}{l}-.1060 \\
p=.164\end{array}$ & $\begin{array}{r}.0161 \\
\mathrm{p}=.833\end{array}$ \\
\hline Mundo de belleza & $\begin{array}{l}-.0372 \\
p=.626\end{array}$ & $\begin{array}{l}-.0648 \\
p=.396\end{array}$ & $\begin{array}{r}.1216 \\
p=.110\end{array}$ & $\begin{array}{r}.1899 \\
\mathrm{p}=.012\end{array}$ \\
\hline Libertad & $\begin{array}{r}.1493 \\
p=.049\end{array}$ & $\begin{array}{l}-.1398 \\
\mathrm{p}=.066\end{array}$ & $\begin{array}{r}.2468 \\
p=.001\end{array}$ & $\begin{array}{r}.1743 \\
\mathrm{p}=.021\end{array}$ \\
\hline Vida excitante & $\begin{array}{r}.2209 \\
p=.003\end{array}$ & $\begin{array}{r}.0065 \\
p=.933\end{array}$ & $\begin{array}{r}.0731 \\
\mathrm{p}=.338\end{array}$ & $\begin{array}{l}-.1989 \\
\mathrm{p}=.009\end{array}$ \\
\hline Valiente & $\begin{array}{r}.1333 \\
p=.079\end{array}$ & $\begin{array}{l}-.1444 \\
p=.057\end{array}$ & $\begin{array}{r}.2146 \\
p=.004\end{array}$ & $\begin{array}{r}.1295 \\
\mathrm{p}=.088\end{array}$ \\
\hline
\end{tabular}


Tabla Il

(Cont.)

\begin{tabular}{|c|c|c|c|c|}
\hline $\begin{array}{l}\text { Valores } \\
\text { terminales }\end{array}$ & $\begin{array}{c}\text { Escala } \\
M\end{array}$ & $\begin{array}{l}\text { Escala } \\
\mathbf{F}\end{array}$ & $\begin{array}{l}\text { Escala } \\
\text { M-F }\end{array}$ & Edad \\
\hline Educado & $\begin{array}{l}-.0686 \\
p=.369\end{array}$ & $\begin{array}{r}.1690 \\
p=.026\end{array}$ & $\begin{array}{l}-.1965 \\
p=.009\end{array}$ & $\begin{array}{r}.0834 \\
p=.274\end{array}$ \\
\hline Intelectual & $\begin{array}{r}.0053 \\
p=.944\end{array}$ & $\begin{array}{l}-.0688 \\
p=.367\end{array}$ & $\begin{array}{r}.1474 \\
\mathrm{p}=.052\end{array}$ & $\begin{array}{r}.1296 \\
\mathrm{p}=.088\end{array}$ \\
\hline Honrado & $\begin{array}{l}-.1109 \\
p=.145\end{array}$ & $\begin{array}{r}.1255 \\
\mathrm{p}=.099\end{array}$ & $\begin{array}{l}-.1394 \\
p=.067\end{array}$ & $\begin{array}{l}-.0605 \\
p=.427\end{array}$ \\
\hline Obediente & $\begin{array}{l}-.1553 \\
p=.041\end{array}$ & $\begin{array}{r}.0258 \\
\mathrm{p}=.736\end{array}$ & $\begin{array}{l}-.1954 \\
p=.010\end{array}$ & $\begin{array}{l}-.1085 \\
\mathrm{p}=.154\end{array}$ \\
\hline Lógico & $\begin{array}{r}.0339 \\
p=.657\end{array}$ & $\begin{array}{l}-.0912 \\
\mathrm{p}=.231\end{array}$ & $\begin{array}{r}.1370 \\
p=.071\end{array}$ & $\begin{array}{r}.1076 \\
\mathrm{p}=.158\end{array}$ \\
\hline Imaginativo & $\begin{array}{r}.0468 \\
p=.540\end{array}$ & $\begin{array}{l}-.0703 \\
p=.356\end{array}$ & $\begin{array}{r}.1099 \\
p=.149\end{array}$ & $\begin{array}{l}-.0704 \\
p=.356\end{array}$ \\
\hline Capaz & $\begin{array}{r}.1540 \\
\mathrm{p}=.043\end{array}$ & $\begin{array}{l}-.1036 \\
p=.174\end{array}$ & $\begin{array}{r}.2130 \\
\mathrm{p}=.005\end{array}$ & $\begin{array}{r}.0070 \\
\mathrm{p}=.927\end{array}$ \\
\hline Alegre & $\begin{array}{l}-.0672 \\
\mathrm{p}=.378\end{array}$ & $\begin{array}{r}.1709 \\
p=.024\end{array}$ & $\begin{array}{l}-.1453 \\
\mathrm{p}=.056\end{array}$ & $\begin{array}{l}-.1010 \\
\mathrm{p}=.185\end{array}$ \\
\hline Autocontrolado & $\begin{array}{l}-.0263 \\
p=.730\end{array}$ & $\begin{array}{r}.0031 \\
p=.968\end{array}$ & $\begin{array}{l}-.0914 \\
p=.230\end{array}$ & $\begin{array}{l}-.0356 \\
p=.641\end{array}$ \\
\hline Capaz de perdonar & $\begin{array}{l}-.1313 \\
\mathrm{p}=.084\end{array}$ & $\begin{array}{r}.1178 \\
p=.122\end{array}$ & $\begin{array}{l}-.1991 \\
\mathrm{p}=.008\end{array}$ & $\begin{array}{l}-.1481 \\
\mathrm{p}=.051\end{array}$ \\
\hline Capaz de amar & $\begin{array}{l}-.0962 \\
\mathrm{p}=.207\end{array}$ & $\begin{array}{r}.0778 \\
p=.308\end{array}$ & $\begin{array}{l}-.0508 \\
p=.505\end{array}$ & $\begin{array}{l}-.1227 \\
\mathrm{p}=.107\end{array}$ \\
\hline Responsable & $\begin{array}{l}-.0970 \\
\mathrm{p}=.203\end{array}$ & $\begin{array}{l}-.0513 \\
\mathrm{p}=.501\end{array}$ & $\begin{array}{l}-.0383 \\
p=.616\end{array}$ & $\begin{array}{r}.0529 \\
\mathrm{p}=.488\end{array}$ \\
\hline Independiente & $\begin{array}{r}.2072 \\
p=.006\end{array}$ & $\begin{array}{l}-.0813 \\
\mathrm{p}=.286\end{array}$ & $\begin{array}{r}.2408 \\
p=.001\end{array}$ & $\begin{array}{l}-.0254 \\
\mathrm{p}=.739\end{array}$ \\
\hline Limpio & $\begin{array}{r}.0454 \\
p=.552\end{array}$ & $\begin{array}{l}-.0140 \\
p=.855\end{array}$ & $\begin{array}{r}-.0829 \\
p=.277\end{array}$ & $\begin{array}{r}.0212 \\
\mathrm{p}=.782\end{array}$ \\
\hline Ambicioso & $\begin{array}{r}.0724 \\
\mathrm{p}=.343\end{array}$ & $\begin{array}{l}-.0901 \\
p=.237\end{array}$ & $\begin{array}{l}-.0148 \\
p=.846\end{array}$ & $\begin{array}{l}-1345 \\
p=.077\end{array}$ \\
\hline Liberal & $\begin{array}{r}.0112 \\
\mathrm{p}=.883\end{array}$ & $\begin{array}{l}-.1681 \\
p=.027\end{array}$ & $\begin{array}{r}.0979 \\
p=.199\end{array}$ & $\begin{array}{r}.2212 \\
\mathrm{p}=.003\end{array}$ \\
\hline Servicial & $\begin{array}{l}-.0754 \\
p=.322\end{array}$ & $\begin{array}{r}.2984 \\
\mathrm{p}=.000\end{array}$ & $\begin{array}{l}-.1494 \\
\mathrm{p}=.049\end{array}$ & $\begin{array}{l}-.0274 \\
\mathrm{p}=.719\end{array}$ \\
\hline
\end{tabular}




\begin{tabular}{|c|c|}
\hline $\begin{array}{c}\text { Escalas } \\
\text { M, F y M-F }\end{array}$ & Edad \\
\hline Masculinidad & $\begin{array}{c}0.639 \\
p=.402\end{array}$ \\
\hline Feminidad & $\begin{array}{c}.0201 \\
\mathrm{p}=.792\end{array}$ \\
\hline $\begin{array}{l}\text { Masculinidad- } \\
\text { feminidad }\end{array}$ & $\begin{array}{c}.1789 \\
\mathrm{p}=.018\end{array}$ \\
\hline
\end{tabular}

en sí mismo, autosuficiencia)», «Capaz (competente, efectivo)», y «Valiente (defender las propias ideas)", en este orden de importancia. Existe, por otra parte, una relación negativa con la variable instrumental «Obediente (deferente, respetuoso)".

La escala de Feminidad, a su vez, correlaciona de forma positiva con "Armonía interna» y de forma negativa con la variable «Libertad", entre los valores terminales. Con respecto a los instrumentales, esta escala se asocia con signo positivo a los siguientes valores en orden creciente de importancia: «Educado», «Alegre (de corazón abierto, gozoso)», y «Servicial (trabaja por el bienestar ajeno)». De forma negativa, las correlaciones significativas se establecen con "Valiente», que aparecía con el signo opuesto en relación a la masculinidad, y "Liberal (abierto de mente)".

La escala de Masculinidad-Feminidad correlaciona de forma positiva con «Libertad», entre los valores terminales. Es oportuno señalar que este valor ha aparecido también significativamente relacionado con las escalas de Masculinidad y Feminidad, positivamente con la primera y con signo negativo con la segunda. De forma negativa la escala M-F correlaciona, por orden, con los valores de "Armonía interna» y «Seguridad familiar (cuidado a los seres queridos)». Interesa destacar que este último valor aparece justamente con signo contrario en relación a la feminidad.

En cuanto a los valores instrumentales, la escala M-F presenta resultados de correlación positiva, en orden de importancia, con los siguientes: «independiente», "Valiente». "Capaz", e "Intelectual», y con signo negativo, también en orden de importancia, con «Capaz de perdonar», «Educado", «Obediente», «Servicial», y "Alegre». De modo muy significativo, puede observarse que los valores «Libertad» y «Armonía interna», entre los terminales, y «Valiente", "Educado», «Obediente», "Capaz», "Alegre», "Independiente", y "Servicial», entre los instrumentales, aparecen con el mismo signo en las escalas $\mathrm{M}$ y $\mathrm{M}-\mathrm{F}$ e igualmente aparecen, pero con signo contrario, en la escala $\mathrm{F}$.

Respecto a la relación de la edad con el autoconcepto de género y el sistema de valores, cabe hacer las observaciones siguientes. El autoconcepto de género sólo en el caso de la escala M-F presenta una correlación significativa con la edad. Entre los valores terminales correlacionan positivamente con la edad "Mundo de belleza», "Igualdad", y «Libertad", en orden de importancia; negativamente se relaciona, también en ese orden con "Vida excitante", "Amistad verdadera (relaciones comunicativas)", "Felicidad (satisfacción)», y "Salvación (sentirse salvado en la vida eterna)». Finalmente, sólo un valor de los instrumentales, «Liberal», correlaciona de 
forma positiva con la edad, en tanto que "Capaz de perdonar (dispuesto a perdonar a los otros)" lo hace negativamente.

\section{Valores discriminantes para sexo y género}

Hemos realizado un análisis factorial, encontrándonos con una puntuación en el test de Kaiser de .06991 que restaba significación a los resultados. Hacía falta un elevado número de factores para explicar tan sólo el $68 \%$ de la varianza, lo que hacía inviable este tipo de análisis. Tan sólo señalar que según nuestros datos la escala $M-F$ va asociada con signo positivo a los valores «Liberal», «Libertad», e "Imaginativo», y de forma negativa a «Obediente».

Un posterior análisis discriminante nos permitió identificar aquellos valores que delimitan, por un lado, el grupo de mujeres del grupo de varones $y$, por otro, a los sujetos categorizados como masculinos de los categorizados como femeninos. Por lo que se refiere al primero de los aspectos, los valores «Seguridad nacional», "Igualdad», «Mundo en paz», "Madurez», y «Armonía interna», entre los terminales y "Honrado», "Autocontrolado», «Educado», «Intelectual», «Lógico», "Liberal», y "Ambicioso", entre los instrumentales, son, por este orden, los que tienen una mayor función diferenciadora. La capacidad de clasificación de las funciones discriminantes obtenidas mediante los valores terminales e instrumentales queda recogida en la Tabla III.

TABLA III

Análisis discriminante del RVS en relación al sexo

\begin{tabular}{|c|c|c|c|c|}
\hline & \multirow{2}{*}{ Grupo real } & \multirow{2}{*}{$\begin{array}{l}\text { Número } \\
\text { de casos }\end{array}$} & \multicolumn{2}{|c|}{ Grupo de predicción } \\
\hline & & & Mujeres & Varones \\
\hline \multirow{2}{*}{ (1) } & Grupo de mujeres & 132 & $\begin{array}{c}92 \\
(69,7 \%)\end{array}$ & $\begin{array}{c}40 \\
(30,3 \%)\end{array}$ \\
\hline & Grupo de varones & 42 & $\begin{array}{c}16 \\
(38,1 \%)\end{array}$ & $\begin{array}{c}26 \\
(61,9 \%)\end{array}$ \\
\hline
\end{tabular}

PORCENTAJE DE CASOS «AGRUPADOS» CLASIFICADOS CORRECTAMENTE: $67,82 \%$

\begin{tabular}{|c|c|c|c|c|}
\hline \multirow{2}{*}{ (2) } & Grupo de mujeres & 132 & $\begin{array}{c}92 \\
(69,7 \%)\end{array}$ & $\begin{array}{c}40 \\
(30,3 \%)\end{array}$ \\
\hline & Grupo de varones & 42 & $\begin{array}{c}13 \\
(31,0 \%)\end{array}$ & $\begin{array}{c}29 \\
(69,0 \%)\end{array}$ \\
\hline & $\begin{array}{l}\text { PORCENTAJE D } \\
\text { CORRECTAMEI }\end{array}$ & S & ICADOS & \\
\hline
\end{tabular}

(1) Valores Terminales.

(2) Valores Instrumentales.

Con respecto a las características psicológicas de masculinidad y feminidad, se obtienen resultados igualmente discriminates: el $73 \%$ de los sujetos femeninos y el $65 \%$ de los masculinos aparecen asi claramente dis- 


\section{3}

criminados, cuando se utilizan los valores terminales, siendo, de mayor a menor, los más discriminativos las variables 9 (armonía interna), 18 (una vida excitante), 10 (madurez), 8 (amistad verdadera), 15 (seguridad nacional), 11 (un mundo en paz) y 13 (reconocimiento social). Cuando se utilizan los valores instrumentales, el porcentaje de casos correctamente clasificados es, en conjunto, de $73 \%$ (ver Tabla IV), apareciendo como más discriminantes, por orden de importancia, las seis variables siguientes: v. 18 (servicial), v. 4 (honrado), v. 9 (alegre), v. 1 (valiente), v. 12 (capaz de amar) y v. 2 (educado).

TABLA IV

Análisis discriminante del RVS en relación al género

\begin{tabular}{|c|c|c|c|c|}
\hline & \multirow{2}{*}{ Grupo real } & \multirow{2}{*}{$\begin{array}{l}\text { Número } \\
\text { de casos }\end{array}$} & \multicolumn{2}{|c|}{ Grupo de predicción } \\
\hline & & & Mujeres & Varones \\
\hline \multirow{3}{*}{ (1) } & Grupo femenino & 138 & $\begin{array}{c}101 \\
(73,2 \%)\end{array}$ & $\begin{array}{c}37 \\
(26,8 \%)\end{array}$ \\
\hline & Grupo masculino & 29 & $\begin{array}{c}10 \\
(34,5 \%)\end{array}$ & $\begin{array}{c}19 \\
(65,5 \%)\end{array}$ \\
\hline & \multicolumn{4}{|c|}{$\begin{array}{l}\text { PORCENTAJE DE CASOS *AGRUPADOS» CLASIFICADOS } \\
\text { CORRECTAMENTE: } 71,86 \%\end{array}$} \\
\hline \multirow{3}{*}{ (2) } & Grupo femenino & 138 & $\begin{array}{c}101 \\
(73,2 \%)\end{array}$ & $\begin{array}{c}37 \\
(26,8 \%)\end{array}$ \\
\hline & Grupo masculino & 29 & $\begin{array}{c}8 \\
(27,6 \%)\end{array}$ & $\stackrel{21}{(72,4 \%)}$ \\
\hline & $\begin{array}{l}\text { PORCENTAJE DE CAS } \\
\text { CORRECTAMENTE: } 7\end{array}$ & ADOS* CI & ICADOS & \\
\hline
\end{tabular}

(1) Valores Terminales.

(2) Valores Instrumentales.

\section{CONCLUSIONES}

Una primera observación importante sugerida por los resultados parece indicar que, en nuestra muestra de universitarios, la condición de sexo no tiende a coincidir, como reiteradamente han puesto de manifiesto numerosos estudios, con la condición psicosexual (masculinidad y feminidad). Significativamente, sí se constata relación entre el sexo y las puntuaciones en la escala de Masculinidad-Feminidad (M-F) del EPAQ. Nuestro análisis se alinea, en este sentido, con las elaboraciones teóricas de Spence y Helmreich (1978) acerca del hecho de que las puntuaciones en las escalas de Masculinidad (M) y Feminidad (F) recogen una serie de rasgos estereotipados de uno y otro sexo, en tanto que la escala de Masculinidad-Feminidad (M-F) estaría integrada por características que son o no deseables socialmente en función del sexo.

Aunque no abundan las investigaciones acerca de los correlatos de valor y los roles sexuales, distintos trabajos previos contraponen los rasgos agente-instrumentales, en los que predomina una orientación asertiva ca- 


\section{4}

racterísticamente masculina, a los aspectos comunicativos y expresivos propios de la feminidad. Feather (1984) destaca los valores de ambición e independencia como predominantes en sujetos con «fuerte esquema masculino", mientras que en los sujetos "esquemáticamente femeninos" sobresalen los relativos al cultivo de las relaciones interpersonales.

En nuestro estudio, los varones conceden importancia a valores como la inteligencia, la lógica y el autocontrol, y a otros de distinto carácter, como la igualdad, mientras que las mujeres lo hacen en relación a la corrección y la honradez, mostrando asimismo su inclinación hacia la armonía interna y la seguridad. Estos resultados nos llevan a participar de la interpretación referida acerca de la asociación entre los rasgos de carácter agente/instrumental y comunal/expresivos y el hecho de ser, respectivamente, varón o mujer. Nuestro trabajo podría incluso abonar la conclusión de que esto mismo es también predicable del sistema de género. Así, hemos visto que la masculinidad se asocia a valores como libertad, vida excitante e independencia, y la feminidad a la armonía interna o al servicio a los demás.

La masculinidad-feminidad se halla asociada a la edad. También, en relación con los valores, se observa con la edad un cambio axiológico, desde un mayor interés por las amistades sinceras, la felicidad, y la ambición, a una disposición a valorar positivamente la belleza, la igualdad y las prácticas liberales.

Tal vez las observaciones más ricas de nuestro estudio han sido las obtenidas tras llevar a cabo los dos análisis discriminantes, con el fin de conocer cuáles son los valores, en su doble modalidad, terminales e instrumentales, que mejor permiten clasificar a los sujetos, de acuerdo con el sexo biológico, por un lado, y en relación al género psicológico, por otro. En este sentido, cabe resaltar el contraste encontrado entre los valores terminales, donde casi todas las variables que aparecen como más discriminantes para el sexo se repiten en el género, aunque modificando el orden (v. 11, $15,10,9)$, y los valores instrumentales, en los que sólo coinciden las variables 4 y 2 , y en este último caso con un orden totalmente distinto.

Podríamos decir que, entre las metas generales de existencia, tanto los varones y las mujeres como los sujetos categorizados masculinos y femeninos aparecen bien discriminados en base a características referidas a una vida tranquila, tal y como reflejan los valores de madurez, seguridad y mundo en paz. Con respecto a modos más concretos de comportamiento y actuación, la honradez y corrección de formas así como la lógica y el autocontrol destacan, cuando se intenta discriminar entre los subgrupos varón y mujer, mientras que la delimitación entre los sujetos masculinos y los femeninos aparece mejor representada por valores relativos al servicio a los demás, capacidad de amar, y al valor en defensa de las propias ideas.

Finalmente, señalar que las medidas de masculinidad y feminidad no resultan ser autodescripciones objetivas, afectivamente neutrales, sino que, en línea con los trabajos de Feather (1984), entre otros, se muestran como evaluaciones prescriptivas de la realidad. Quizás habría que concluir, de nuevo con Feather (1978), que más que utilizar medidas generales de masculinidad y feminidad, factorialmente complejas y multidimensionales, podría tener sentido servirse de rasgos interpersonales independientes, derivados empíricamente. 


\section{Referencias}

ANASTASI, A. (1985). Reciprocal relations between cognitive and affective development with implications for sex differences. En T. B. Sonderegger (comp.). Nebraska Symposium on Motivation 1984: Psychology and Sender. University of Nebraska Press, Lincoln/Londres.

AtKinson, J. W. (1964). An introduction to motivation. Van Nostrand, Priceton, Nueva Jersey.

Barbera, E. (1983). Psicología de la mujer y roles sexuales. Psicológica, 4, 49-65.

BARBERA, E y MAYOR, L. (1987). Consideraciones sobre la motivación de logro y el autoconcepto de género. Boletín de Psicología. En prensa.

BEM, S. L. (1981). Gender schema theory: A cognitive account of sex typing. Psichological Rezuiew, 88, 354-364.

COHEN, J. y COHEN, P. (1975). Applied multiple regression/correlation analysis for the behavioral sciences. Hillsdale, Nueva Jersey. Erlbaum.

CRANE, M. y MARKUS, H. (1982). Identidad de género: las ventajas de una aproximación al autoesquema. En I. Martínez (comp.). Aportaciones a la medida de los constructos de género. Lecturas básicas. Universidad de Valencia, Valencia.

De VEGA, M. (1984). Introducción a la psicología cognitiva. Alianza, Madrid.

FEATHER, N. T. (1971). Organization and discrepancy in cognitive structures. Psychological Review, 78, 355-379.

Feather, N. T. (1975). Values in education and society. Free Press, Nueva York.

FEATHER, N. T. (1978). Factor structure of the Bem Sex-Role Inventory: Implications for the study of masculinity, femininity, and androgyny. Australian Journal of Psychology, 30, 241-254.

FEATHER. N. T. (1984). Masculinity, femininity, psychological androgyny, and the structure of values. Journal of Personality and Social Psychology, 47, 604-620.

FERNÁNDEZ, J. (1983). Nuevas perspectivas en la medida de la masculinidad y feminidad. Editorial de la Universidad Complutense, Madrid.

GARCÉS, J. (1985). Sistema de valores en la política autonómica: perfil ideológico de discursos políticos. Tesis doctoral. Valencia.

HAMILTON, D. L. (1981). Cognitive representations of persons. En E. T. Higgins, C. P. Herman y M. P. Zanna (comp.). Social cognition, the Ontario symposium, 1. L.E.A. Hillsdale, Nueva Jersey.

HAYS, W. L. (1967). Quantification in psychology. Belmont, CA; Brooks/Cole.

Higgins, E. T.; HeRMAan, C. P. y ZanNA, M. P. (1981). Social cognition. The Ontario Symposium, 1. L.E.A. Hillsdale, Nueva Jersey.

HORNER, M. (1968). A psychological barrier to achievement in women: The motive to avoid success. Simposio de la Midwestern Psychological Association. En D. C. McClelland y R. S. Steele (comp.). Human motivation. A book of readings (1973). General Learning Press, Morristown (Nueva Jersey).

Markus, H.; CRANE, M.; BERNSTEIN, S. y Siladi, M. (1982). Self-schemas and gender. Journal of Personality and Social Psychology, 42, 38-50.

ROKEACH, M. (1973). The nature of human values. Free Press: Nueva York.

Rumelhart, D. E. y OrTONY, A. (1977). The representation of knowledge in memory. En R. C. Anderson; R. Spiro $y$ W. E. Montagne (comp.). Schooling and the acquisition of knowledge. L.E.A. Hillsdale, Nueva Jersey.

SPENCE, J. T. y HELMREICH, R. L. (1978). Masculinity and femininity: Their psychological dimensions, correlates, and antecedents. Austin: University of Texas Press.

SPenCE, J. T.; Helmreich, R. L. y Holahan, C. K. (1979). Negative and positive components of psychological masculinity and femininity, and their relationship to self-reports of neurotic and acting out behaviors. Journal of Personality and Social Psychology, 37, 1673-168. 